\title{
DISTORTIONS DUE TO THE SLIDING MICROTOME
}

\author{
WILFRID TAYLOR DEMPSTER \\ Department of Anatomy, University of Michigan, Ann Arbor \\ ONE FIGURE
}

The foregoing paper on the mechanics of sectioning and a study of the extent and characteristics of section distortion by the rotary microtome (Dempster, '42; ' 43 , in press) have dealt in some detail with the distorting effects of the sectioning reaction for the transverse knife. Extension of the analysis to certain reactions in oblique cutting by the sliding microtome reveal general relationships that pertain to both the sliding and the rotary microtome.

The rotary microtome has a knife axis set at $90^{\circ}$ to the axis of block movement, whereas in the sliding microtome, the knife is ordinarily fixed obliquely to the axis of movement. When a knife is set obliquely, a greater length of knife edge and a greater effective area of the outer facet of a knife are employed in sectioning.

An imaginary plane, simultaneously parallel to the axis of knife movement and perpendicular to the block face of the material being sectioned, may be visualized as intersecting the outer bevel of a knife in the sliding microtome. If the knife is at a right angle to the axis of movement, i.e., comparable to the position in the rotary microtome, the imaginary plane intersects the outer bevel at a smaller rake angle ${ }^{1}$ than if the knife were set obliquely. Similarly, if the obliquity of a knife is changed from a transverse position to a position where its edge coincides with the axis of knife movement, the

\footnotetext{
1 The rake angle or normal rake of a knife is the angle, in a plane transverse to a knife edge, between the outer beveled facet and the perpendicular to the new block surface.
} 
rake angle in the axis of knife movement increases to a maximum of $90^{\circ}$. A knife with an obliquity of $45^{\circ}$ has the same rake angle directed to the side as it does anteriorly. As the knife set is made more nearly transverse to the axis of movement, the side rake increases and the anterior or front rake decreases toward a minimum value; conversely, side rake decreases and front rake increases as the knife obliquity approaches the axis of movement. Some effect may be postulated as due to this unsymmetrical relationship of front and side rakes.

If a $90^{\circ}$ knife passes almost across the face of an elongate block of paraffin in the cutting of a section, the knife may be backed away in order to leave the partly detached section with the block. The section may then be flattened on the block surface with brushes. There is no displacement to either side and no overhanging of section edges. The free end of the section, however, depending upon the degree of section compression, is more or less displaced from the adjacent end of the block.

Now, if the same block is sectioned with an oblique knife, and the section is similarly flattened, the section is both distorted and reoriented. Furthermore, relationships vary depending upon the angle of obliquity to which the knife is set. Figure 1 depicts the shape and extent of displacement of sections of the same thickness for knife sets of $15^{\circ}, 30^{\circ}$ and $45^{\circ}$, and a normal rake, in each case, of $55^{\circ}$. In all, the section outline is rhomboidal rather than rectangular as was the block face. The sections are wider than the block (greatest widths are associated with the $45^{\circ} \mathrm{knife}$ ), and the long axis of the section is displaced laterally. For the $15^{\circ} \mathrm{knife}$, displacement is to the left (away from the knife), while for the $30^{\circ}$ and $45^{\circ}$ knives, the sections slant to the right (or toward the knife) and this slant is more for the $45^{\circ}$ than for the $30^{\circ}$ knife.

Likewise, there is an increased compression in the axis of the knife movement as the knife angle is increased toward $45^{\circ}$. For knife angles greater than $45^{\circ}$, the amount of com- 
pression decreases and approaches values dealt with in the study on the rotary microtome. Lateral displacement to the right becomes negligible at about $70^{\circ}$, and then begins a slight deviation to the left similar to that figured with the $15^{\circ} \mathrm{knife}$. As the knife obliquity angle increases from $45^{\circ}$ to $90^{\circ}$ or decreases toward $0^{\circ}$, section width decreases to that of the original block. These effects are by no means limited to

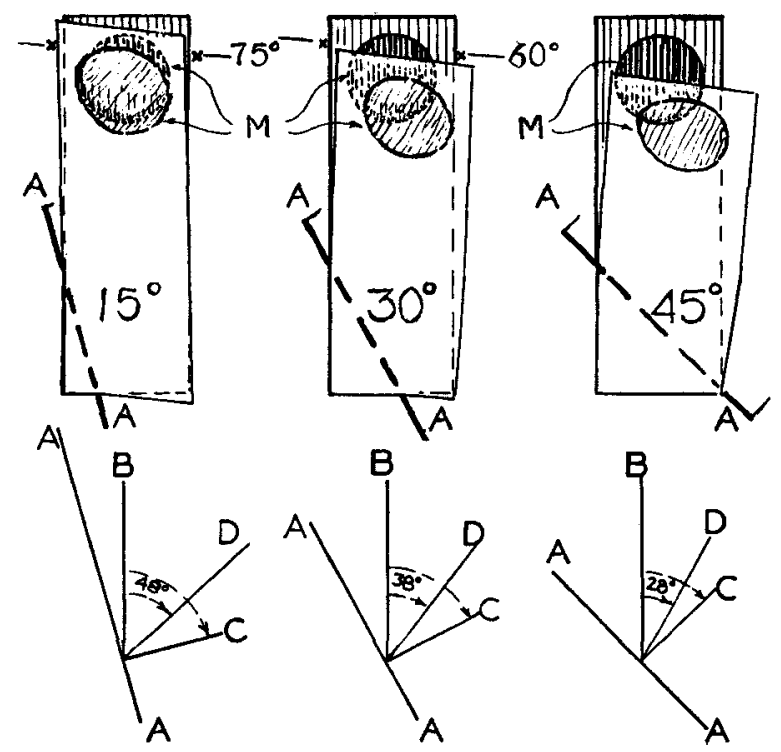

Fig. 1 The upper diagrams show distortions of sections by the sliding microtome for characteristic knife obliquities as indicated by $\mathrm{A}-\mathrm{A}$. " $\mathrm{M}$ " represents both the face of a cylinder of celloidin embedded in the block (vertical shading) and the distorted shape appearing in the section. Lines labeled $60^{\circ}$ and $75^{\circ}$ indicate the relative amount of section shortening in similarly shaped sections for $60^{\circ}$ and $75^{\circ}$ knife obliquity. Figures below show the significant axes involved in sectioning with the $15^{\circ}, 30^{\circ}$ and $45^{\circ}$ knife. $A-A=$ knife edge; $B=$ axis of movement; $\mathrm{C}=$ cross sectional axis of knife; $\mathrm{D}=$ axis of compression.

paraffin sectioning; they may be shown with celloidin and in fact may be duplicated roughly with a hand plane and a block of soft wood such as pine or balsa.

Four effects thus appear as characteristic of the action of an oblique knife: (1) compression in the cutting axis which reaches a maximum for a $45^{\circ} \mathrm{knife}$ and then decreases to a 
standard compression at $90^{\circ} ;(2)$ increased section width which also becomes maximal at $45^{\circ} ;(3)$ a lateral deviation of section axis toward the knife from about $20^{\circ}$ to $70^{\circ}$ (being maximal at $45^{\circ}$ ) and away from the knife for angles in the range $20^{\circ}$ to $0^{\circ}$ and $70^{\circ}$ to $90^{\circ}$; and (4) increased section thickness to compensate for decreased section area. For thin sections, both lateral displacement and compression are greater than for thick sections.

In order to determine the direction of the axis of elongation (i.e., increased section width) and also the true compression axis, as contrasted with compression in the axis of knife movement, lathe-turned cylinders of paraffin infiltrated celloidin, colored, were re-embedded and sectioned. After such sections were flattened and affixed to glass plates, a magnified image of each was projected in an Edinger projector. The celloidin slice appeared as an elliptical or possibly egg-shaped area having perceptible parallel straight scorings due to microscopic imperfections of the knife edge. These scorings, also evident on the block surface with oblique illumination, corresponded with the direction of knife movement. By determining the major and minor axes of the ellipsoidal slices, it was possible to determine also the angles between these axes and the axis of knife movement. Thus, data were provided which could be visualized in terms of knife obliquity and direction of knife movement. The appearance of celloidin slices is represented on the diagrams already discussed (fig. 1 at M).

The smaller the knife angle, the farther was the axis of section compression displaced from the axis of knife movement. Conversely, as knife obliquity was increased toward $90^{\circ}$ the axis of compression came closer to and finally, at $90^{\circ}$, coincided with the axis of knife movement. The axis of section elongation was, of course, $90^{\circ}$ further removed. Elongation, when compared with compression, appeared to be of no great magnitude. In all cases, the compression axis is directed toward rather than away from the knife, and it falls between the axis of movement and the axis of knife cross section. Moreover, if the angle lying between the axis of movement 
and the compression axis is compared with the angle between the axis of movement and the axis of maximum facet angle, a constant ratio obtains. For a knife so tilted that the upper facet makes an angle of $35^{\circ}$ with the block face (i.e., normal rake of $55^{\circ}$ ), the former angle is close to $63.5 \%$ of the latter. These relationships, too, are diagrammed for $15^{\circ}, 30^{\circ}$ and $45^{\circ} \mathrm{knife}$ angles at the lower part of figure 1 .

Though the amount of section shortening varies, as indicated above, with obliquity, a knife set at an angle of $20^{\circ}$, an angle commonly used, caused only a third the amount of compression of sections that was found with the rotary microtome (i.e., at similar section thickness and similar normal rake). Although compression was less, warping distortions, not possible with the rotary microtome, were present.

Except for the altered axes of distortion, the mechanies of section formation are the same for the oblique knife as for the transverse knife (preceding paper). Tissues are deformed like celloidin cylinders or like the paraffin itself, but the extent of deformation is ordinarily somewhat less than for the matrix material. The distortions, likewise, are permanent and may be significant in certain types of microscopy.

Correction of compression defects may be desirable in the making of drawings and plastic or graphic reconstructions. For sections from the rotary microtome, the one dimensional compression defect, if the increased section thickness is ignored, may be compensated for by: (1) transforming to a square system of coordinates, projection drawings made on a rectangular grid system with proportions corresponding with the gross distortion, (2) projection of the image of a section to an oblique drawing board and drawing the image as each level of the board is brought into focus, and (3) the use of appropriate cylinder lenses added to a projector. With a biaxial distortion in the plane of focus, however, as for sections from the sliding microtome. either the photographic method of Heard ('31), or the transference of a drawing from an appropriate rhomboidal grid system to a square system is required. 


\section{LITERATURE CITED}

DEMPSTER, W. T. 1942 The mechanics of paraffin sectioning by the microtome. Anat. Rec., vol. 84, pp. 241-268.

Parafin compression due to the rotary microtome. Stain Tech., vol. 18, (in press).

HEARD, O. O. 1931 A photographic method of orienting serial sections for reconstruction. Anat. Ree., vol. 49 , pp. 59-70. 